\title{
Estimativa da acidez potencial através do método do pH SMP em solos de altitude de Santa Catarina
}

\author{
Marcos Gervasio Pereira' ${ }^{1}$ André Lucas Simões Araújo², Denilson Dortzbach³ \\ Orlando Carlos Huertas Tavares ${ }^{4}$ e Eduardo Carvalho da Silva Neto ${ }^{5}$
}

\begin{abstract}
Resumo - A correção do pH de solos ácidos é de suma importância para o aumento da produtividade das culturas, sendo a calagem uma das práticas mais usuais para essa correção. Nos estados de Rio Grande do Sul e Santa Catarina, essa recomendação tem como base a determinação do pH do solo com o emprego da solução tampão SMP. Esse método vem sendo utilizado nesses estados devido a sua praticidade e aplicação para o cálculo de calagem. Entretanto, é preciso que haja regionalização desta estimativa devido às diferentes características físicas, químicas e mineralógicas dos solos. $\mathrm{O}$ objetivo do estudo foi avaliar as relações entre o pH SMP, os valores de $\mathrm{H}+\mathrm{Al}$ e os atributos químicos do solo, carbono orgânico total e fracionamento químico da matéria orgânica. Foram utilizadas 43 amostras de horizontes superficiais de solos de altitude de Santa Catarina. A partir dos resultados, pode-se verificar que o pH SMP apresenta uma correlação significativa com o teor de $\mathrm{H}+\mathrm{Al}$, assim como outros atributos químicos dos solos. Dessa forma, é possível estimar a acidez potencial por intermédio do modelo exponencial $62,48 \mathrm{e}^{-0,41 \mathrm{pHSMP} \text {. }}$
\end{abstract}

Termos para indexação: Alumínio; análise química; calagem; capacidade de troca catiônica.

\section{Estimation of potential acidity by the pH SMP method in soils of altitude from Santa Catarina}

Abstract - Correction of the $\mathrm{pH}$ of acid soils is critical to increase crop productivity and liming is one of the most common practices for this correction. In Rio Grande do Sul and Santa Catarina states, this recommendation is based on soil pH determination using the SMP buffer solution. This method has been used in these states due to its practicality and application for liming calculation. However, this estimate must be regionalized due to the different physical, chemical and mineralogical characteristics of the soils. The objective of this study was to evaluate the relationship between pH SMP, H + Al content and soil chemical attributes, total organic carbon and chemical fractionation of organic matter. Forty-three samples of surface horizons of highland soils of Santa Catarina were used in this study. From the results, it can be verified that the SMP pH presents a significant correlation with the $\mathrm{H}+\mathrm{Al}$ content, as well as other chemical attributes of the soils. Thus it is possible to estimate the potential acidity through the exponential model $62,48 \mathrm{e}^{-0,41 \mathrm{pHSMP}}$, and the values of $\mathrm{H}+\mathrm{Al}$ can be estimated by this equation.

Index terms: Aluminum; cation exchange capacity; chemical analysis; liming.

\section{Introdução}

Os solos brasileiros, em sua maioria, são naturalmente ácidos, com necessidade de correção do $\mathrm{pH}$ para que as culturas tenham melhor aproveitamento dos nutrientes e maior potencial produtivo (MASCARENHAS et al., 2007). Nos estados de Santa Catarina e Rio Grande do Sul, o método de recomendação de calagem que se destaca é por meio da solução-tampão SMP, que estima a acidez potencial bem como a exigência da calagem nos solos. Escosteguy \& Bissani
(1999) e Kaminskiet et al. (2002) analisaram o uso da solução-tampão SMP para solos da Região Sul do Brasil, e verificaram que este método apresenta boa correlação com a extração de $\mathrm{H}+\mathrm{Al}$ pelo extrator acetato de cálcio, comprovando sua eficiência.

Nos solos de regiões de altitude, as condições climáticas favorecem o acúmulo de matéria orgânica e o predomínio de $\mathrm{Al}^{3+}$ no complexo sortivo. Dessa forma, tornam-se necessárias recomendações de calagem específicas, baseadas na utilização de doses elevadas de calcário que podem acelerar o processo de mineralização da matéria orgânica.

Pereira et al. (1998) e Silva et al. (2006) observaram, por exemplo, que o teor de carbono orgânico foi o atributo químico que mais influenciou na acidez potencial dos Organossolos. Portanto, em se tratando de solos que apresentam elevados teores de matéria orgânica e $\mathrm{Al}$, devem ser testados métodos diferenciados para a recomendação da calagem, pois esses atributos podem contribuir para a não quantificação dos valores de acidez de forma adequada.

Recebido em 2/10/2019. Aceito para publicação em 21/3/2020.

${ }^{1}$ Engenheiro-agrônomo, Dr., Universidade Federal Rural do Rio de Janeiro (UFRRJ), BR 465 km 7, Depto de Solos, 23897-000 Seropédica, RJ, fone (21) 37873772, e-mail: mgervasiopereira01@gmail.com.

${ }^{2}$ Estudante de Agronomia, UFRRJ, e-mail: andre23.araujo@yahoo.com.br

${ }^{3}$ Engenheiro-agrônomo, Dr. Empresa de Pesquisa Agropecuária e Extensão Rural de Santa Catarina/ Ciram, Av Admar Gonzaga, 1347, Itacorubi, 88034-901, Florianópolis, SC, fone (48) 36655135, e-mail:denilson@epagri.sc.gov.br

${ }^{4}$ Engenheiro-agrônomo, Dr. (UFRRJ), e-mail: ochtavares@gmail.com

${ }^{5}$ Engenheiro-agrônomo, M.Sc. (UFRRJ), e-mail: netocseduardo@gmail.com 
Assim, o objetivo deste estudo foi obter uma equação de regressão, bem como o coeficiente de correlação, capaz de estimar os teores de $\mathrm{H}+\mathrm{Al}$ através do $\mathrm{pH}$ da solução tampão ( $\mathrm{pH}$ SMP) em solos de regiões de altitude do estado de Santa Catarina. Adicionalmente, foram avaliadas relações do $\mathrm{pH}$ SMP com os valores de $\mathrm{pH}$ em água, $\mathrm{pH}$ em $\mathrm{KCl}$, disponibilidade de $\mathrm{Ca}, \mathrm{Mg}, \mathrm{K}, \mathrm{P}$ e $\mathrm{Al}$ no solo, carbono orgânico total (COT) e frações da matéria orgânica de amostras de horizontes superficiais desses solos.

\section{Material e métodos}

O estudo foi desenvolvido com perfis de solos localizados nas regiões de Água Doce, Campos Novos e São Joaquim no estado de Santa Catarina (Tabela 1). As regiões do presente estudo localizam-se em áreas cuja altitude varia entre 849 a 1359 m, e os solos são formados por litologia predominante de rochas efusivas, com predomínio de riodacitos (ácidos) e basalto (básicos). Alguns perfis também se localizam em áreas cujo material de origem são rochas sedimentares gonduânicas paleozoicas correspondentes à Bacia do Paraná (MAACK, 2001).

De acordo com a classificação climática de Köppen, o clima nas regiões amostradas é identificado como Cfb (clima subtropical com verões brandos) e o mês mais quente apresenta temperatura média inferior a $22^{\circ} \mathrm{C}$. A vegetação é composta por Florestas Subtropicais, com destaque para a Floresta de Araucária (Floresta Ombrófila Mista) que transpõe as serras costeiras e se estende pelo Planalto Catarinense. Os campos do Planalto (Campos de Altitude) aparecem como áreas isoladas, entremeadas à Floresta de Araucária, onde predominam gramíneas de baixo e médio porte, além de ciperáceas, verbenáceas, leguminosas e compostas (SANTA CATARINA, 1986).

Nas áreas de estudo foram abertas trincheiras para coleta de 43 perfis modais descritos segundo Santos et al. (2013a). A partir dos atributos morfológicos e dos dados de análises físicas e químicas, os perfis de solo foram classificados segundo o Sistema Brasileiro de Classificação de Solos (SiBCS) (SANTOS et al., 2013b). Os perfis foram identifica- dos como: Nitossolo Háplico, Nitossolo Vermelho, Nitossolo Bruno, Latossolo Vermelho, Cambissolo Húmico, Cambissolo Háplico e Neossolo Litólico (Tabela $1)$.

Para a coleta das amostras foi utilizado somente o horizonte superficial dos perfis. Após a coleta as amostras foram secas ao ar, destorroadas e passadas por peneira $(2 \mathrm{~mm})$, obtendo-se assim a terra fina seca ao ar (TFSA). Na TFSA foram avaliados os seguintes atributos químicos e físicos: $\mathrm{pH}$ em água, $\mathrm{pH}$ em $\mathrm{KCl}, \mathrm{Ca}^{2+}, \mathrm{Mg}^{2+}, \mathrm{K}^{+}$, soma de bases (SB), $\mathrm{Al}^{3+}, \mathrm{H}+\mathrm{Al}$, capacidade de troca de cátions a $\mathrm{pH} 7,0$ (CTC pH 7,0), saturação por bases $(\mathrm{V})$, saturação por alumínio (m) e a textura, quantificando-se as frações areia, silte e argila pelo método da pipeta (DONAGEMMA et al., 2011). A determinação da acidez total com solução tampão SMP foi realizada conforme Raij \& Quaggio (1983). O fracionamento das substâncias húmicas (SHs) foi avaliado em triplicata nas 43 amostras dos horizontes superficiais. Foram determinados os teores de carbono das frações humina (HM), ácidos húmicos $(\mathrm{AH})$ e ácidos fúlvicos (AF) segundo a técnica de solubilidade diferencial estabelecida pela Sociedade Internacional de SHs (SWIFT, 1996), com adaptações (BENITES et al., 2003).
Foram realizadas análises de correlação linear simples entre os valores de $\mathrm{H}+\mathrm{Al}$, pH SMP, pH em $\mathrm{H}_{2} \mathrm{O}, \mathrm{pH}$ em $\mathrm{KCl}$ das 43 amostras de solo, sendo indicada a equação de melhor ajuste em conformidade com o coeficiente de determinação pelo teste $\mathrm{F}$ a $5 \%$ de probabilidade. Adicionalmente foi realizada uma matriz de correlação multivariada, associando todas as características químicas das amostras dos horizontes estudados. As análises foram feitas com o auxílio do Software Microsoft Office Excel 2007.

\section{Resultados e discussão}

Os valores de $\mathrm{pH}$ SMP variaram de 3,53 a 7,20 e a acidez potencial $(\mathrm{H}+\mathrm{Al})$ entre 2,95 e 18,59 (Tabela 2). Verificase que, com o aumento da concentração de $\mathrm{H}+\mathrm{Al}$, houve a diminuição do pH SMP. A relação encontrada entre os valores de $\mathrm{H}+\mathrm{Al}$ e $\mathrm{pH}$ SMP apresentou correlação exponencial negativa $\left(R^{2}=-0,86^{* *}\right)$ descrita pela equação $Y$ $=62,48 \mathrm{e}^{-0,41 x}$ (Figura 1). Constatou-se correlação positiva moderada $\left(R^{2}=0,5\right.$ a $0,7)$ entre o $\mathrm{pH}$ em água e o $\mathrm{pH}$ em $\mathrm{KCl}$ com o pH SMP (Tabela 3).

O padrão verificado nesse estudo é concordante com o observado por Pereira et al. (1998), que analisaram 41 ,

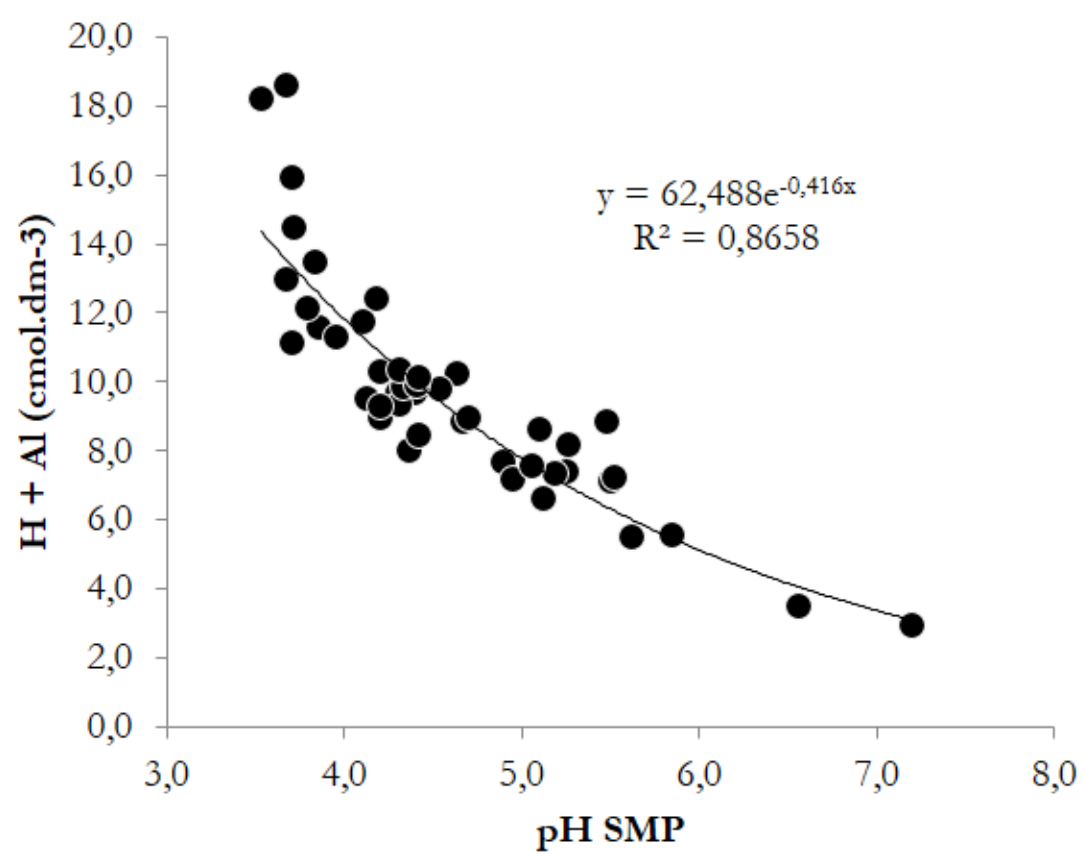

Figura 1. Relação entre acidez potencial (H + Al) e o pH SMP em amostras coletadas em regiões de altitude de SC

Figure 1. Relationship between potential acidity $(H+A l)$ and $p H S M P$ in samples collected in high altitude regions of SC 
Tabela 1. Localização, altitude e classificação dos solos estudados Table 1. Location, height and classification of the studied soils

\begin{tabular}{|c|c|c|c|c|}
\hline \multirow{2}{*}{$\begin{array}{l}\text { Perfil } \\
\text { no }\end{array}$} & \multicolumn{2}{|c|}{ Coordenadas* } & \multirow{2}{*}{$\begin{array}{l}\text { Atitude } \\
\text { (m) }\end{array}$} & \multirow[t]{2}{*}{ Classificação } \\
\hline & $\mathbf{x}$ & y & & \\
\hline 1 & 641606 & 6908000 & 912 & CAMBISSOLO HÁPLICO Alítico típico \\
\hline 2 & 648336 & 6929661 & 902 & CAMBISSOLO HÁPLICO Alítico típico \\
\hline 3 & 634183 & 6928696 & 864 & CAMBISSOLO HÁPLICO Ta Distrófico léptico \\
\hline 4 & 640564 & 6909346 & 1.144 & CAMBISSOLO HÚMICO Alítico típico \\
\hline 5 & 624991 & 6930230 & 1.014 & CAMBISSOLO HÁPLICO Alítico típico \\
\hline 6 & 692137 & 6933879 & 1.059 & CAMBISSOLO HÁPLICO Alítico típico \\
\hline 7 & 614882 & 6887112 & 1.258 & NITOSSOLO BRUNO Distrófico húmico \\
\hline 8 & 614804 & 6886861 & 1.282 & NEOSSOLO LITÓLICO Distrófico típico \\
\hline 9 & 614952 & 6887292 & 1.259 & NITOSSOLO BRUNO Alumínico típico \\
\hline 10 & 617122 & 6886388 & 1.359 & CAMBISSOLO HÁPLICO Tb Distrófico típico \\
\hline 11 & 616935 & 6888630 & 1.259 & CAMBISSOLO HÁPLICO Alítico típico \\
\hline 12 & 601323 & 6872431 & 1.304 & CAMBISSOLO HÁPLICO Alítico típico \\
\hline 13 & 602781 & 6874099 & 1.274 & CAMBISSOLO HÁPLICO Ta Distrófico léptico \\
\hline 14 & 601649 & 6874949 & 1.240 & CAMBISSOLO HÁPLICO Tb Distrófico típico \\
\hline 15 & 602748 & 6876005 & 1.227 & CAMBISSOLO HÁPLICO Tb Distrófico típico \\
\hline 16 & 598834 & 6876373 & 1.302 & CAMBISSOLO HÁPLICO Alítico típico \\
\hline 17 & 599164 & 6876935 & 1.301 & CAMBISSOLO HÁPLICO Tb Distrófico típico \\
\hline 18 & 598479 & 6880683 & 1.270 & CAMBISSOLO HÁPLICO Tb Distrófico típico \\
\hline 19 & 600111 & 6874767 & 1.328 & CAMBISSOLO HÁPLICO Tb Distrófico típico \\
\hline 20 & 591146 & 6876251 & 1.112 & NITOSSOLO HÁPLICO Distrófico típico \\
\hline 21 & 587203 & 6878623 & 1.146 & CAMBISSOLO HÁPLICO Tb Distrófico típico \\
\hline 22 & 595322 & 6892674 & 1.109 & CAMBISSOLO HÁPLICO Tb Distrófico típico \\
\hline 23 & 593195 & 6856252 & 1.217 & CAMBISSOLO HÁPLICO Alítico típico \\
\hline 24 & 607759 & 6867101 & 1.312 & CAMBISSOLO HÁPLICO Alítico típico \\
\hline 25 & 525172 & 6939550 & 900 & NITOSSOLO VERMELHO Distrófico típico \\
\hline 26 & 507146 & 6925654 & 989 & CAMBISSOLO HÁPLICO Alítico típico \\
\hline 27 & 419723 & 7015409 & 1183 & NITOSSOLO VERMELHO Alítico típico \\
\hline 28 & 484770 & 6953453 & 869 & NITOSSOLO HÁPLICO Distrófico típico \\
\hline 29 & 483745 & 6975319 & 976 & NITOSSOLO HÁPLICO Alumínico típico \\
\hline 30 & 472186 & 6955375 & 877 & LATOSSOLO VERMELHO Distrófico típico \\
\hline 31 & 470390 & 6961938 & 852 & LATOSSOLO VERMELHO Distrófico típico \\
\hline 32 & 477681 & 6962361 & 884 & NITOSSOLO VERMELHO Distrófico típico \\
\hline 33 & 477522 & 6971137 & 949 & CAMBISSOLO HÁPLICO Tb Distrófico típico \\
\hline 34 & 494783 & 6969905 & 880 & NITOSSOLO HÁPLICO Distrófico típico \\
\hline 35 & 482296 & 6992408 & 912 & NITOSSOLO VERMELHO Alumínico típico \\
\hline 36 & 488274 & 6989440 & 1.162 & CAMBISSOLO HÁPLICO Ta Distrófico típico \\
\hline 37 & 456679 & 6950047 & 858 & CAMBISSOLO HÁPLICO Tb Distrófico típico \\
\hline 38 & 487706 & 6988949 & 1.055 & NITOSSOLO HÁPLICO Eutrófico típico \\
\hline 39 & 485194 & 7006662 & 919 & NITOSSOLO HÁPLICO Distrófico típico \\
\hline 40 & 488210 & 7009152 & 849 & CAMBISSOLO HÚMICO Alítico típico \\
\hline 41 & 448263 & 7030119 & 1.330 & CAMBISSOLO HÚMICO Alítico típico \\
\hline 42 & 450148 & 7045289 & 1.260 & CAMBISSOLO HÁPLICO Alítico típico \\
\hline 43 & 456577 & 7045197 & 1.260 & CAMBISSOLO HÁPLICO Alítico típico \\
\hline
\end{tabular}

amostras de horizontes superficiais de solos com elevado teor de matéria orgânica de vários estados do Brasil. Os resultados demonstraram que a acidez potencial pode ser estimada por meio da regressão da solução-tampão SMP ( $\mathrm{r}$ $\left.=0,85^{* *}\right)$.

Escosteguy \& Bissani (1999) estudaram a relação entre o valor de $\mathrm{H}+\mathrm{Al}$ e o $\mathrm{pH}$ SMP, visando estabelecer uma equação para a estimativa do valor de $\mathrm{H}$ + Al (acidez potencial), o qual pode ser utilizado no cálculo da CTC (método da soma de cátions trocáveis em amostras de solo) dos estados de Santa Catarina e Rio Grande do Sul. Os autores também verificaram uma relação entre os valores de $\mathrm{H}+\mathrm{Al}$ e o $\mathrm{pH}$ de SMP, que pode ser descrita de forma significativa $\left(R^{2}=\right.$ $0,90)$ pela equação exponencial $\mathrm{H}+\mathrm{Al}=$ 7968,4e-0,90045mp.

Há uma correlação positiva forte dos valores de $\mathrm{pH}$ SMP com disponibilidade de Ca e Mg e negativa forte com $\mathrm{H}$ + Al. Tanto para a fração ácido fúlvico (AF) quanto para a fração ácido húmico os maiores valores de correlação foram verificados com o valor de T, o que indica que essas frações estão contribuindo para o aumento das cargas presentes nos coloides do solo.

As correlações lineares dos valores do $\mathrm{pH}$ SMP com os de $\mathrm{pH}\left(\mathrm{H}_{2} \mathrm{O}\right)$ e $\mathrm{pH}$ (KCl) foram positivas $\left(\mathrm{R}^{2}=0,60 * * / \mathrm{R}^{2}=\right.$ $0,59^{* *}$ ) respectivamente, sendo ambas classificadas como moderadas (Tabela 3).

Os valores de $\mathrm{H}+\mathrm{Al}$ estimados pelo o modelo matemático proposto nesse estudo, quando comparados, são mais baixos. Esse padrão pode ser decorrente das características dos solos, em especial em função dos elevados teores de carbono verificados nos horizontes estudados (PEREIRA et al., 1998).

Os resultados evidenciam a importância do ajuste da equação de acordo com a região, em virtude das variações dos atributos químicos, físicos e mineralógicos dos solos. Através da equação $62,48 \mathrm{e}^{-0,41 \text { pHSMP }}$ é possível estimar a acidez potencial com precisão considerável para as amostras de horizontes superficiais dos solos de altitude de Santa Catarina, com os valores de $\mathrm{pH}$ SMP na faixa avaliada neste trabalho (Tabela 5). 
Tabela 2. Caracterização químicas das amostras dos horizontes superficiais

Table 2. Chemical characterization of samples from the superficial horizons

\begin{tabular}{|cccccc|}
\hline Atributo & Média & Mediana & Mínimo & Máximo & Desvio-Padrão \\
\hline $\mathrm{pH}\left(\mathrm{H}_{2} \mathrm{O}\right)$ & 5,03 & 5,06 & 4,02 & 5,88 & 0,45 \\
\hline $\mathrm{pH}(\mathrm{SMP})$ & 4,61 & 4,40 & 3,53 & 7,20 & 0,79 \\
\hline $\mathrm{H}+\mathrm{Al}\left(\mathrm{cmol}_{\mathrm{c}} \mathrm{dm}^{-3}\right)$ & 9,70 & 9,42 & 2,95 & 18,59 & 3,20 \\
\hline $\mathrm{Ca}^{2+}\left(\mathrm{cmol}_{\mathrm{c}} \mathrm{dm}^{-3}\right)$ & 2,60 & 2,19 & 0,10 & 6,90 & 2,01 \\
\hline $\mathrm{Mg}^{2+}\left(\mathrm{cmol}_{\mathrm{c}} \mathrm{dm}^{-3}\right)$ & 2,69 & 2,50 & 0,40 & 7,40 & 1,80 \\
\hline $\mathrm{K}^{+}\left(\mathrm{cmol}_{\mathrm{c}} \mathrm{dm}^{-3}\right)$ & 1,30 & 0,85 & 0,20 & 4,70 & 1,09 \\
\hline $\mathrm{Al}^{3+}\left(\mathrm{cmol}_{\mathrm{c}} \mathrm{dm}^{-3}\right)$ & 2,24 & 1,89 & 0,20 & 7,00 & 1,83 \\
\hline $\mathrm{S}\left(\mathrm{cmol}_{\mathrm{c}} \mathrm{dm}^{-3}\right)$ & 6,65 & 6,27 & 1,00 & 16,40 & 4,10 \\
\hline $\mathrm{COT}\left(\mathrm{gkg}^{-1}\right)$ & 26,48 & 25,23 & 5,00 & 16,9 & 13,11 \\
\hline Humina $\left(\mathrm{g} \mathrm{kg}^{-1}\right)$ & 12,52 & 10,66 & 1,31 & 33,25 & 7,48 \\
\hline Ácido Fúlvico $(\mathrm{FAF} \mathrm{gkg})$ & 6,60 & 6,30 & 1,55 & 12,53 & 2,41 \\
\hline Ácido Húmico $(\mathrm{FAH} \mathrm{gkg}$ & \\
\hline
\end{tabular}

Tabela 3. Matriz de correlação de Pearson entre os diferentes métodos de medição do pH e a fertilidade do solo e frações das substâncias húmicas dos diferentes tipos de solos de altitude de Santa Catarina

Table 3. Pearson's correlation matrix between different $\mathrm{pH}$ measurement methods and soil fertility and fractions of humic substances from different types of soils in Santa Catarina

\begin{tabular}{|c|c|c|c|c|c|c|c|c|c|c|c|c|c|c|c|}
\hline & $\mathrm{pHH}_{2} \mathrm{O}$ & $\mathrm{pHKCl}$ & $\mathrm{Ca}$ & $\mathrm{Mg}$ & K & $\mathrm{Na}$ & $P$ & Al & $\mathrm{H}+\mathrm{Al}$ & $S$ & $T$ & Humina & AF & $\mathrm{AH}$ & СOT \\
\hline pH SMP & 0,60 & 0,59 & 0,86 & 0,81 & 0,19 & 0,27 & $-0,04$ & $-0,82$ & $-0,86$ & 0,83 & 0,66 & $-0,07$ & $-0,11$ & $-0,11$ & 0,06 \\
\hline $\mathrm{pH} \mathrm{H} \mathrm{H}_{2} \mathrm{O}$ & & 0,99 & 0,57 & 0,60 & 0,00 & 0,18 & 0,09 & $-0,62$ & $-0,70$ & 0,54 & 0,37 & $-0,09$ & 0,11 & 0,09 & 0,04 \\
\hline $\mathrm{pH} \mathrm{KCl}$ & & & 0,55 & 0,56 & $-0,04$ & 0,19 & 0,09 & $-0,61$ & $-0,69$ & 0.50 & 0,33 & $-0,10$ & 0,10 & 0,09 & 0,03 \\
\hline $\mathrm{Ca}$ & & & & 0,87 & 0,27 & 0,27 & 0,12 & $-0,83$ & $-0,73$ & 0,95 & 0,83 & $-0,11$ & $-0,11$ & $-0,14$ & 0,04 \\
\hline $\mathrm{Mg}$ & & & & & 0,19 & 0,25 & 0,20 & $-0,75$ & $-0,71$ & 0,92 & 0,83 & $-0,01$ & $-0,06$ & $-0,14$ & 0,05 \\
\hline K & & & & & & 0,24 & 0,30 & -0.22 & $-0,10$ & 0,49 & 0,56 & $-0,14$ & $-0,10$ & $-0,30$ & 0,16 \\
\hline $\mathrm{Na}$ & & & & & & & 0,48 & $-0,21$ & $-0,14$ & 0,31 & 0,31 & $-0,10$ & 0,14 & 0,13 & 0,04 \\
\hline$P$ & & & & & & & & 0,00 & 0,14 & 0.23 & 0,32 & 0,10 & 0,28 & 0,19 & 0,19 \\
\hline Al & & & & & & & & & 0,83 & -0.79 & $-0,50$ & 0,02 & 0,06 & 0,03 & 0.00 \\
\hline $\mathrm{H}+\mathrm{Al}$ & & & & & & & & & & $-0,70$ & $-0,47$ & 0,17 & 0,21 & 0,17 & 0,17 \\
\hline$S$ & & & & & & & & & & & 0,92 & $-0,10$ & $-0,11$ & $-0,21$ & 0,04 \\
\hline $\mathrm{T}$ & & & & & & & & & & & & $-0,13$ & $-0,12$ & $-0,28$ & 0,06 \\
\hline Humina & & & & & & & & & & & & & 0,67 & 0,63 & 0,85 \\
\hline $\mathrm{AF}$ & & & & & & & & & & & & & & 0,88 & 0,87 \\
\hline $\mathrm{AH}$ & & & & & & & & & & & & & & & 0,79 \\
\hline
\end{tabular}

Em negrito, valores significativos em nível $\alpha=0,050$ (teste bilateral). Ácido fúlvico (AF), ácido húmico (AH), Carbono orgânico total (COT ). 
Tabela 4. Estimativa dos teores de H + Al no solo para valores de pH SMP na faixa de 4,5 a 7,0 por meio de equações ajustadas para diferentes estados e regiões brasileiras

Table 4. Estimation of $\mathrm{H}+\mathrm{Al}$ levels in the soil for $\mathrm{pH}$ SMP values in the range of 4.5 to 7.0 using adjusted equations for different Brazilian states and regions

\begin{tabular}{|c|c|c|c|c|c|c|c|}
\hline \multicolumn{8}{|c|}{ Valores estimados de $\mathrm{H}+\mathrm{Al}\left(\mathrm{cmol}_{\mathrm{c}} \mathrm{dm}^{-3}\right)$} \\
\hline \multirow{2}{*}{ Estado ou Região } & \multicolumn{6}{|c|}{ pH SMP } & \multirow{2}{*}{ Referência } \\
\hline & 4,5 & 5,0 & 5,7 & 6,0 & 6,8 & 7,0 & \\
\hline São Paulo & 20,52 & 12,12 & 5,80 & 4,23 & 1,82 & 1,48 & Quaggio et al. (1985) \\
\hline Rio de Janeiro & 23,51 & 14,12 & 6,91 & 5,09 & 2,25 & 1,84 & Pereira et al. (1998) \\
\hline Rio Grande do Sul e SC & 13,86 & 8,83 & 4,70 & 3,59 & 1,75 & 1,46 & Escosteguy \& Bissani (1999) \\
\hline Pernambuco & 10,06 & 8,11 & 5,80 & 4,95 & 3,11 & 2,75 & Nascimento (2000) \\
\hline Nordeste & 23,15 & 15,53 & 7,52 & 5,04 & 1,19 & 0,85 & Silva et al. (2000) \\
\hline Nordeste Paraense & 14,77 & 11,00 & 6,84 & 5,45 & 2,93 & 2,56 & Gama et al. (2002) \\
\hline Norte de Minas Gerais & 17,29 & 10,49 & 5,21 & 3,86 & 1,74 & 1,42 & Silva et al. (2002) \\
\hline Noroeste Paranaense & 8,27 & 6,95 & 5,09 & 4,30 & 2,18 & 1,65 & Sambatti et al. (2003) \\
\hline Amazonas & 13,33 & 11,41 & 8,71 & 7,56 & 4,48 & 3,71 & Moreira et al. (2004) \\
\hline Vale do Jequitinhonha & 24,55 & 13,99 & 6,37 & 4,55 & 1,85 & 1,47 & Silva et al. (2006) \\
\hline Brejo Paraibano & 21,94 & 14,80 & 7,72 & 5,73 & 3,47 & 3,60 & Chaves et al. (2007) \\
\hline Santa Catarina & 9,87 & 8,04 & 6,04 & 5,34 & 3,85 & 3,54 & Presente trabalho \\
\hline
\end{tabular}

Tabela 5. Estimativa dos valores da acidez potencial $(H+A l)$ através da equação ajustadas $y=62,48 e^{-0,41 p H S M P}$ para os solos de regiões de altitude de SC

Table 5. Estimation of the potential acidity values $(H+A l)$ using the adjusted equation $y=62.48 e^{-0.41 p H S M P}$ for the soils of SC altitude regions

\begin{tabular}{|cccc}
\hline pH SMP & $\begin{array}{c}\mathbf{H}+\mathbf{A l} \\
\mathbf{c m o l} \mathbf{d m}^{-3}\end{array}$ & pH SMP & $\begin{array}{c}\mathbf{H}+\mathbf{A l} \\
\mathbf{c m o l}_{\mathbf{c}} \mathbf{d m}^{-3}\end{array}$ \\
\hline 3,53 & 14,69 & 4,41 & 10,24 \\
\hline 3,67 & 13,87 & 4,42 & 10,20 \\
\hline 3,67 & 13,87 & 4,54 & 9,71 \\
\hline 3,70 & 13,71 & 4,63 & 9,36 \\
\hline 3,70 & 13,70 & 4,67 & 9,20 \\
\hline 3,72 & 13,59 & 4,70 & 9,09 \\
\hline 3,79 & 13,20 & 4,90 & 8,37 \\
\hline 3,83 & 12,99 & 4,95 & 8,20 \\
\hline 3,86 & 12,83 & 5,06 & 7,84 \\
\hline 3,95 & 12,37 & 5,10 & 7,72 \\
\hline 4,10 & 11,63 & 5,12 & 7,65 \\
\hline 4,13 & 11,49 & 5,19 & 7,44 \\
\hline 4,18 & 11,25 & 5,25 & 7,25 \\
\hline 4,20 & 11,16 & 5,26 & 7,23 \\
\hline 4,20 & 11,16 & 5,48 & 6,60 \\
\hline 4,20 & 11,16 & 5,50 & 6,55 \\
\hline 4,30 & 10,71 & 5,52 & 6,49 \\
\hline 4,31 & 10,67 & 5,62 & 6,23 \\
\hline 4,31 & 10,67 & 5,85 & 5,67 \\
\hline 4,33 & 10,58 & 6,56 & 4,24 \\
\hline 4,36 & 10,45 & 7,20 & 3,26 \\
\hline
\end{tabular}




\section{Conclusões}

Verifica-se correlação negativa e significativa entre os teores de $\mathrm{H}+\mathrm{Al}$ e o valor do $\mathrm{pH} \mathrm{SMP} \mathrm{e} \mathrm{pH}\left(\mathrm{H}_{2} \mathrm{O}\right), \mathrm{pH}(\mathrm{KCl})$ e os teores de $\mathrm{Ca}$ e $\mathrm{Mg}$ no solo.

$A$ acidez potencial pode ser determinada pela equação $62,48 \mathrm{e}^{-0,41 \mathrm{pHSMP}}$ para os horizontes superficiais dos solos de altitude de Santa Catarina.

\section{Referências}

BENITES, V. M.; MADARI, B.; MACHADO, P. L. O. A. Extração e fracionamento quantitativo de substâncias húmicas do solo: Um procedimento simplificado de baixo custo. Rio de Janeiro: Embrapa Solos, 2003, 7p. (Circular Técnica, 16).

CHAVES L.G.; CHAVES I.B.; VASCONCELOS C.F. Estimativa da acidez potencial pelo método do pH SMP em solos da microrregião homogênea Brejo Paraibano. Revista Brasileira de Engenharia Agrícola e Ambiental, v.11, p.398-403, 2007. DOI: http://dx.doi. org/10.1590/S1415-43662007000400009.

DONAGEMA, G. K; CAMPOS, D. V. B; CALDERANO S. B; TEIXEIRA, W. G; VIANA, J. H. $M$. Manual de métodos de análise do solo. Rio de Janeiro: Embrapa Solos, 2011. 225p.

ESCOSTEguY, P. A.; BISSANI, C. A. ESTIMATIVA DE $\mathrm{H}+\mathrm{AL}$ pelo $\mathrm{pH}$ SMP em solos do Rio Grande do Sul e de Santa Catarina. Revista Brasileira de Ciência do Solo, Viçosa, v.23, p.175-179. 1999. DOI: http://dx.doi. org/10.1590/S0100-06831999000100021.

GAMA, M.A.P.; PROCHNOW, L.I.; GAMA, J.R.N.F. Estimativa da acidez potencial pelo método SMP em solos ocorrentes no nordeste Paraense. Revista Brasileira de Ciência do Solo, Viçosa, v.26, p.1093-1097, 2002. DOI: http://dx.doi.org/10.1590/S010006832002000400027.

MAACK, R. Breves notícias sobre a geologia dos estados do Paraná e Santa Catarina. Brazilian Archives of Biology and Technology. Jubilee: p.169-288, 2001.

MASCARENHAS, H.A.A.; TANAKA, R.T.;
GALLO, P.B.; PEREIRA, J.C.V.N.A.; AMBROSANO G.M.B.; CARMELLO, Q.A.C. Efeito da calagem sobre a produtividade de grãos, óleo e proteína em cultivares precoces de soja. Revista Brasileira de Ciência do Solo, Viçosa, v.31, p.1475-1485, 2007. DOI: $\quad$ http://dx.doi.org/10.1590/s010390161996000100024.

MOREIRA, A.; ALMEIDA, M.P.; COSTA, S.G.; SANTOS, L.S. Acidez potencial pelo método do pH SMP no Estado do Amazonas. Pesquisa Agropecuária Brasileira, Brasília, v.39, p.89-92, 2004. DOI: http://dx.doi. org/10.1590/S0100-204X2004000100013.

NASCIMENTO, C.W.A. Acidez potencial estimada pelo $\mathrm{pH}$ SMP em solos do Estado de Pernambuco. Revista Brasileira de Ciência do Solo, Viçosa, p.24, p.679-682, 2000. DOI: $\quad$ http://dx.doi.org/10.1590/S010006832000000300021.

PEREIRA, M. G.; EBELING, A. G.; VALLADARES, G. S.; ANJOS, L. H. C.; ESPÍNDULA JÚNIOR, A. Estimativa da acidez potencial pelo método do $\mathrm{pH}$ SMP em solos com elevado teor de matéria orgânica. Bragantia, Campinas, v.65, p.487-493, 2006. DOI: $\quad$ http://dx.doi.org/10.1590/S000687052006000300015.

QUAGGIO, J. A.; RAIJ, B.V.; MALAVOLTA, E. Alternative use of the SMP-buffer solution to determine lime requirement of soils. Communications in Soil Science and Plant Analysis, v.16, p.245-260. 1985. DOI: http:// dx.doi.org/10.1080/00103628509367600.

RAIJ, B.VAN., QUAGGIO, J.A. Métodos de análise de solo para fins de fertilidade. Campinas, Instituto Agronômico de Campinas, 1983. 31p. (Boletim Técnico,81)

SAMBATTI, J. A.; SOUZA JUNIOR, I. G.; COSTA, A. C. S.; TORMENA, C. A. Estimativa da acidez potencial pelo método do $\mathrm{pH}$ SMP em solos em formação Caiuá - Noroeste do Estado do Paraná. Revista Brasileira de Ciência do Solo, Viçosa, v.27, p.257- 264, 2003. DOI: $\quad$ http://dx.doi.org/10.1590/S010006832003000200006.

SANTA CATARINA - GABINETE DE PLANEJAMENTO E COORDENAÇÃO GERAL, SUBCHEFIA DE ESTATÍSTICA, GEOGRAFIA E INFOR-
MÁTICA. Atlas de Santa Catarina. Florianópolis. 1986. 173p.

SANTOS, R. D.; LEMOS, R. C.; SANTOS, H. G.; KER, J. C.; ANJOS, L. H. C.; SHIMIZU, S. $H$. Manual de descrição e coleta de solo no campo. Viçosa: Sociedade Brasileira de Ciência do Solo, 100p. 2013a.

SANTOS, H. G. DOS; JACOMINE, P. K. T.; ANJOS, L. H. C. DOS; OLIVEIRA, V. A. DE; LUMBRERAS, J. F.; COELHO, M. R.; ALMEIDA, J. A. DE; CUNHA, T. J. F.; OLIVEIRA, J. $B$. de. Sistema brasileiro de classificação de solos. 3. ed. rev. e ampl. Brasília, DF: Embrapa, 353p. 2013b.

SILVA, C. A.; AVELLAR, M. L.; BERNARDI, A. C. C. Estimativa da acidez potencial pelo $\mathrm{pH}$ SMP em solos do Semi-árido do Nordeste brasileiro. Revista Brasileira de Ciência do Solo, Viçosa 24: 689-692, 2000. DOI: $\quad$ http://dx.doi.org/10.1590/S010006832000000300023.

SILVA, E. B.; DIAS, M. S. C.; GONZAGA, E. I. C.; SANTOS, N. M. Estimativa da acidez potencial pelo $\mathrm{pH}$ SMP em solos da região Norte do Estado de Minas Gerais. Revista Brasileira de Ciência do Solo, Viçosa, v.26, p.561565, 2002. DOI: http://dx.doi.org/10.1590/ S0100-06832002000200031.

SILVA, E.B; COSTA, H.A.O; FARNEZI, M.M. Acidez potencial estimada pelo método do $\mathrm{pH}$ em solos da região do Vale do Jequitinhonha no Estado de Minas Gerais. Revista Brasileira de Ciência do Solo, Viçosa, v.30, p.751-7, 2006

SWIFT, R.S. Organic matter characterization. In: Sparks, D.L.; PAGE, A.L.; HELMKE, P.A.; LOEPPERT, R.H.; SOLTANPOUR, P.N.; TABATABAI, M.A.; JOHNSTON, C.T.; SUMNER, M.E. (Eds.) Methods of soil analysis: chemical methods. vol. 3. Soil Science Society of America; American Society of Agronomy, Madison. (SSSA. BookSeries, 5). p. 10111020. 1996

VETTORI, L. Métodos de análise do solo. Equipe de Pedologia e Fertilidade do Solo, Ministério da Agricultura, Rio de Janeiro, Brasil. 1969. 24p. 